\title{
Desmoplastic fibroma of ilium
}

\author{
Lin-lin Zhang MD, Hui-lin Yang MD, Xue-feng Li MD, Jing Yuan MD \\ Department of Orthopaedics, The First Affiliated Hospital of SooChow University, SooChow, China
}

Key words: Desmoplastic fibroma; Diagnosis; Ilium; Therapy

\section{Introduction}

Desmoplastic fibroma of bone, a rare, benign but locally aggressive tumor, accounts for less than $0.1 \%$ of all primary bone tumors and typically affects adolescent and young adults ${ }^{1,2}$. It is considered the bony counterpart of the much more common desmoid tumors of soft tissue. The long tubular bones are most often involved ${ }^{3,4}$. Here we report a case of desmoplastic fibroma of the right ilium in a 49-year-old female patient and describe its clinical, radiological, and pathological features.

\section{Case report}

A 49-year-old female patient presented to our hospital with an abnormal finding in her right ilium after a conventional radiographic examination. She was able to walk normally and experienced no discomfort. Physical examination showed mild tenderness of her right ilium. LaboStarting export: ratory findings were all within normal limits. A plain X-ray film showed a large osteolytic bony lesion with a well-demarcated margin in the wing of the right ilium. Delicate and diffuse trabeculation with an expansile appearance was observed (Fig. 1). CT demonstrated a lytic expansile lesion with a thin rim and remnant trabeculae in her right ilium (Figs 2, 3). The characteristic radiographic findings and clinical signs suggested this was a benign iliac tumor. Preoperative diagnosis included osteofibrous dysplasia, nonossifying fibroma and desmoplastic fibroma of bone.

After surgical exposure the mass was found to be yellowish-white and to have a rubbery consistency on macroscopic examination. The bone surrounding the lesion was sclerotic and some serous fluid was observed (Fig. 4). A quick preliminary pathological examination during the operation showed proliferation of blandlooking spindle cells in abundant collagenous stroma and

Address for correspondence Hui-lin Yang, MD, Department of Orthopaedics, The First Affiliated Hospital of SooChow University, SooChow, China 215006 Tel: 0086-0512-67780111; Fax: 00860512 67780999; Email: zhangdoublelin@126.com

Received 1 January 2011; accepted 18 February 2011

DOI: 10.1111/j.1757-7861.2011.00136.x

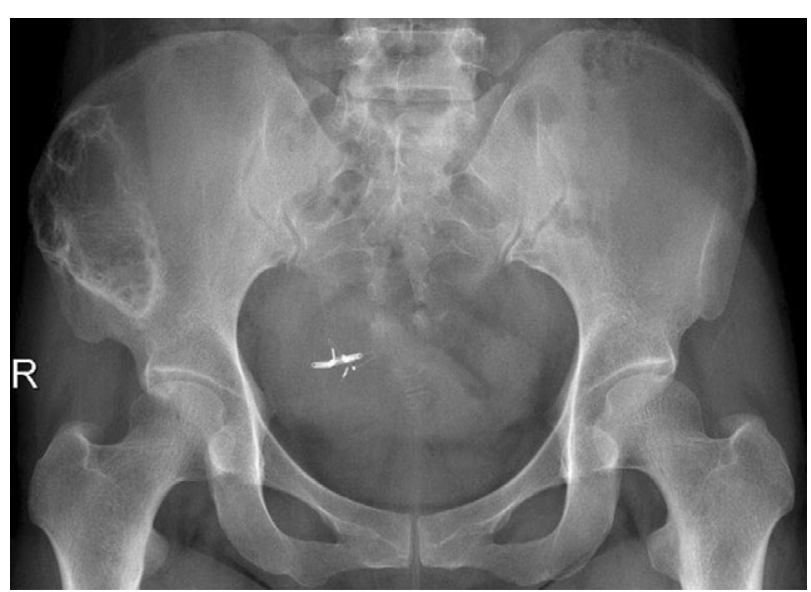

Figure 1 Anteroposterior radiograph of the pelvis showing a large osteolytic bony lesion with a well-defined margin and diffuse trabeculation in the wing of the right ilium.

a provisional diagnosis of desmoplastic fibroma of the right ilium was made. Wide resection of the lesions was advocated. Pathological examination after surgery confirmed a typical desmoplastic fibroma of ilium with slender fibroblasts surrounded by collagen fibers in wavy and interlacing bundles (Fig. 5).

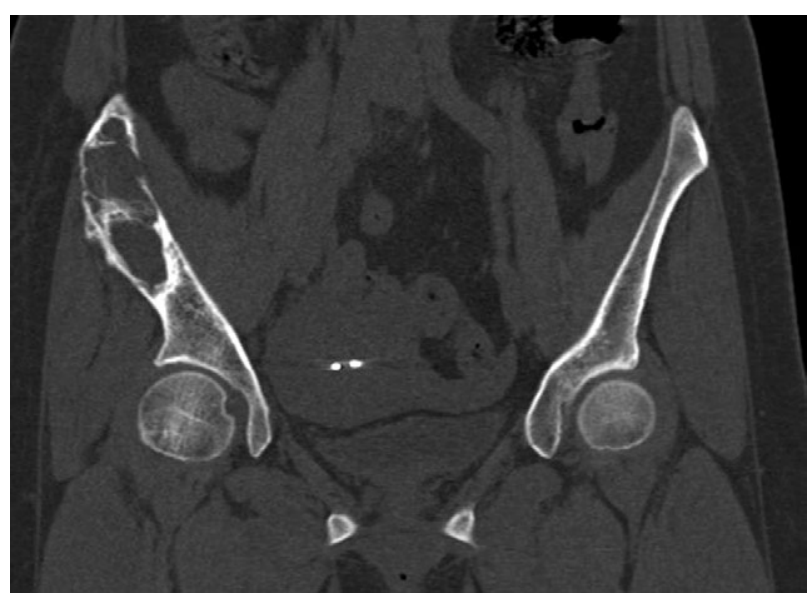

Figure 2 Coronal reconstruction of CT film of the pelvis clearly showing a lytic expansile lesion with a thin rim in the right ilium. 


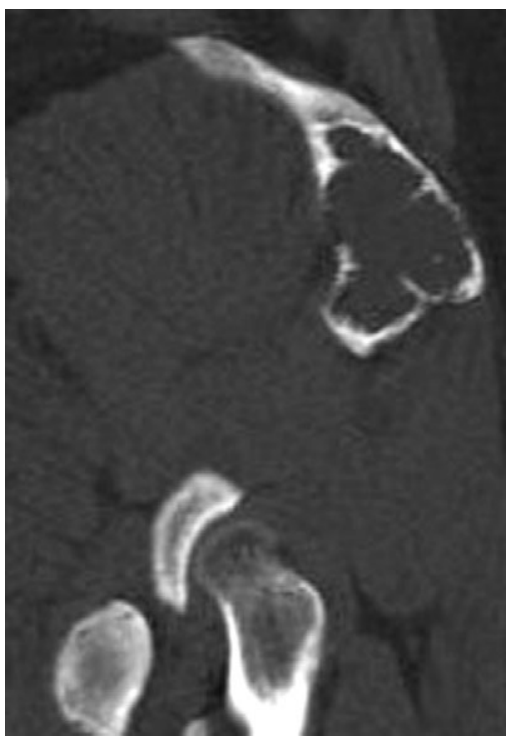

Figure 3 Sagittal reconstruction of Ct film of the ilium revealing remnant trabeculae in the expansible lesion.

\section{Discussion}

Desmoplastic fibroma of bone is a very rare, fibrogenic, benign primary bone tumor characterized by local recurrence after simple curettage and was first described by Jaffe in $1958^{5}$. It has been reported in all age groups, but is more common in the second and third decades ${ }^{6}$. The meta-epiphyseal regions of the tubular bones are most often involved. Pain and swelling are the commonest presenting symptoms ${ }^{1,6,7}$. The classical radiographic appearance is that of an expansile and osteolytic bony lesion with well-defined margins. Delicate and diffuse trabeculation with an expansile appearance is typically visible on plain radiography ${ }^{8}$. CT scan shows a wellcircumscribed low density area with a thin cortex or,

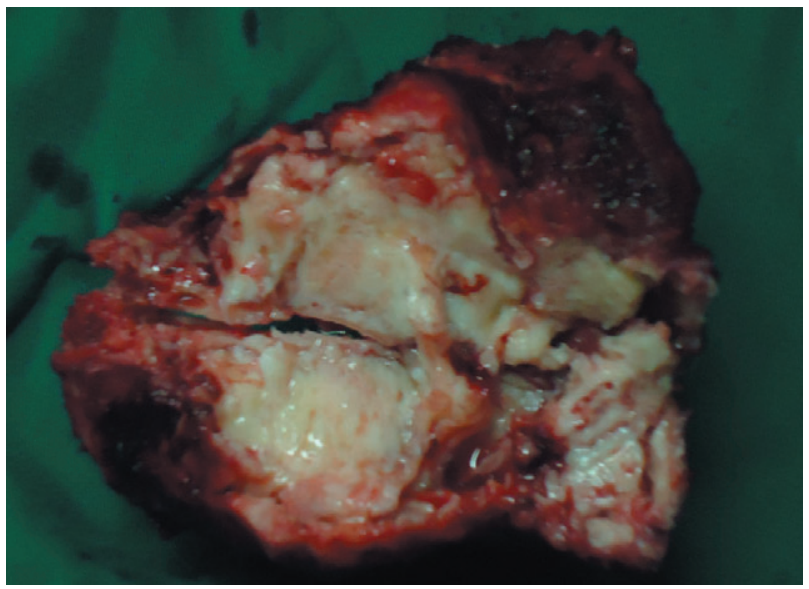

Figure 4 Grossly, the mass is well demarcated and yellowish-white with a rubbery consistency.

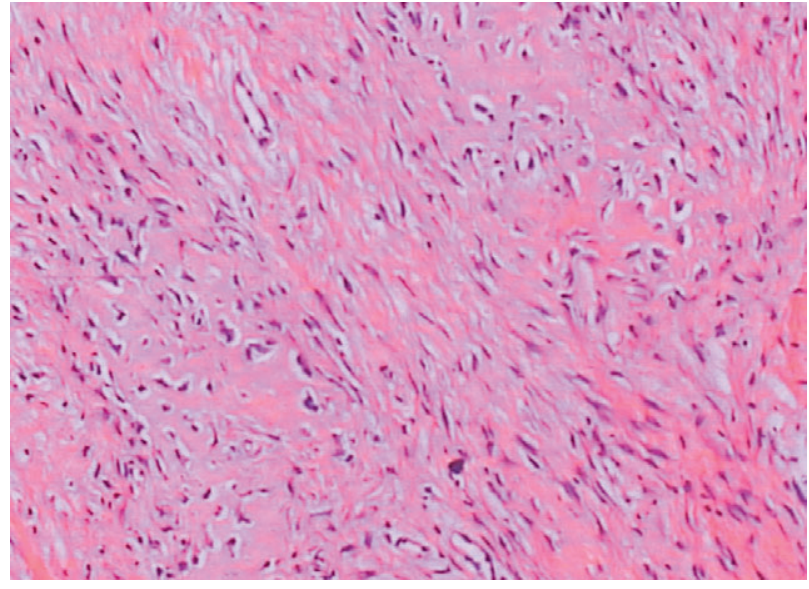

Figure 5 Bundles of spindle cells with abundant intercellular collagen are visible on pathological examination (hematoxylin and eosin $\times 100$ )

sometimes, destruction of the overlying cortex. On MRI desmoplastic fibroma, like other fibrous tumors, may show low signal intensity on $\mathrm{T} 1$ - and T2-weighted images. Grossly, the tissue of the lesion is grayish- or yellowish-white and dense, resembling soft-tissue desmoid lesions. It also resembles these lesions microscopically; being hypocellular and fibroblastic and containing bland-looking spindle cells in abundant collagenous stroma ${ }^{10}$.

As for the histological differential diagnosis, desmoplastic fibroma of bone needs to be distinguished from fibrosarcoma, malignant fibrous histiocytoma, nonossifying fibroma and fibrous dysplasia. A lack of mitotic figures, hypercellularity and pleomorphism help to distinguish this tumor from malignant tumors such as fibrosarcoma and malignant fibrous histiocytoma. Fibroblastic proliferation with abundant cellularity and giant cells and foam cells are usually seen in nonossifying fibroma. Absence of osteoid tissue formation differentiates desmoplastic fibroma from fibrous dysplasia ${ }^{6,711}$.

Wide resection is the treatment of choice for this benign but locally aggressive bone tumor. Jun et al. reported that treatment with aggressive curettage could achieve clinical and radiographic control of this tumor ${ }^{8}$. They reported no recurrences in five patients who underwent aggressive curettage, the follow-up periods ranging from 5 years 6 months to 9 years. Adjuvant treatments have been reported to be effective for treating soft-tissue desmoid tumors (e.g., radiation, anti-inflammatory agents, and cytotoxic agents). Altsmann et al. reported that the recurrence rate of fibromatosis can be reduced significantly by adjuvant radio-therapy ${ }^{12}$, but few data support these approaches as solitary treatment for desmoids tumors in bone. 
In conclusion, desmoplastic fibroma of bone is a rare fibrogenic bone tumor characterized by local aggression and recurrence after simple curettage. An expansile and osteolytic bony lesion with well-defined margins and diffuse trabeculation can be seen on radiographic images. Pathological examination is important in diagnosing this rare entity and excluding other lytic bone lesions. As for therapy, surgical resection is the treatment of choice and periodic follow-up is advocated.

\section{Disclosure}

The authors declare that there are no competing interests in this research.

\section{References}

1. Dorfman H, Czerniak B. Bone tumors. Philadelphia: Mosby, 1998; 514-529.

2. Fletcher C, Unni KK, Mertens F. Pathology and genetics of tumours of soft tissue and bone. Lyon: IARC, 2002; 288.

3. Forest M. Desmoplastic fibroma of bone. In Forest M., Orthopedic surgical pathology: diagnosis of tumors and pseudotumoral lesions of bones and joints. Edinburgh: Churchill Livingstone, 1997; 303-309.
4. Mirra JM, Picci P, Gold RM. Clinical, radiological and pathologic correlations. Philadelphia: Lea \& Febiger, 1989; 735.

5. Jaffe HL. Desmoplastic fibroma and fibrosarcoma. In Jaffe HL., Tumors and tumorous conditions of bones and joints. Philadelphia: Lea \& Febiger, 1958; 298303.

6. Thirupathi RG, Vuletin JC, Wadwa R, et al. Desmoplastic fibroma of the ulna. A case report. Clin Orthop Relat Res, 1983, 179: 231-238.

7. Huvos AG. Bone Tumors, diagnosis, treatment and prognosis. Philadelphia: W.B. Saunders, 1979; 243.

8. Nishida J, Tajima K, Abe $M$, et al. Desmoplastic fibroma. Aggressive curettage as a surgical alternative for treatment. Clin Orthop Relat Res, 1995, 320: 142148.

9. Yu JS, Lawrence S, Pathria $M$, et al. Desmoplastic fibroma of the calcaneus. Skeletal Radiol, 1995, 24: 451-454.

10. Bulychova IV, Unni KK, Bertoni F, et al. Fibrocartilagenous mesenchymoma of bone. Am J Surg Pathol, 1993, 17: 830-836.

11. Inwards CY, Unni KK, Beabout JW, et al. Desmoplastic fibroma of bone. Cancer, 1991, 68: 1978-1983.

12. Altmann S, Lenz-Scharf $O$, Schneider W. Therapeutic options for aggressive fibromatosis. Handchir Mikrochir Plast Chir, 2008, 40: 88-93. 